\title{
NEURITIS ÓPTICA DESMIELINIZANTE TRAS TRATAMIENTO CON INFLIXIMAB
}

\author{
RETROBULBAR OPTIC NEURITIS ASSOCIATED WITH \\ INFLIXIMAB
}

\author{
BIDAGUREN A ${ }^{1}$, MÜLLER-THYSSEN A ${ }^{1}$, BLANCO A ${ }^{1}$, MENDICUTE $\mathrm{J}^{2}$, ÚBEDA M ${ }^{1}$
}

\begin{abstract}
RESUMEN
Caso clínico: Se describe el caso de una paciente de 76 años de edad que presenta una disminución brusca de la agudeza visual en su ojo izquierdo. El cuadro es compatible con una neuritis óptica retrobulbar que en el contexto de una paciente de esta edad y en tratamiento con Infliximab por una artritis reumatoide sugiere la posibilidad de que se trate de una desmielinización asociada al tratamiento con Infliximab.

Discusión: Los inhibidores del TNF $\alpha$ (entre ellos el Infliximab) pueden dar lugar a eventos desmielinizantes entre los cuales puede encontrarse una neuritis óptica retrobulbar.
\end{abstract}

Palabras clave: Neuritis óptica, Infliximab, inhibidores del TNFo, esclerosis múltiple, desmielinización.

\begin{abstract}
Case report: We report the case of a 76-year-old woman who attended our hospital because of a sudden loss of visual acuity in her left eye. The problem appeared to be that of retrobulbar optic neuritis. However, the age of the patient together with the simultaneous use of Infliximab to treat her rheumatoid arthritis, suggests the demyelination may have been associated with the Infliximab use.

Discussion: $\mathrm{TNF} \alpha$ inhibitors (including Infliximab) are associated with demyelinating diseases including optic neuritis (Arch Soc Esp Oftalmol 2007; 82: 109-112).
\end{abstract}

Key words: Optic neuritis, Infliximab, TNF $\alpha$ inhibitors, multiple sclerosis, demyelination.

Recibido: 23/5/06. Aceptado: 13/2/07

Servicio de Oftalmología. Hospital Donostia. San Sebastián (Guipúzcoa). España.

1 Licenciado en Medicina.

2 Doctor en Medicina.

Los autores manifiestan que no tienen interés comercial ni han recibido apoyo económico.

Correspondencia:

Aritz Bidaguren Urbieta

Paseo Dr. Marañón, $14,3{ }^{\circ} \mathrm{C}$

20009 San Sebastián (Guipúacoa)

España

E-mail: aritzbidaguren@kaixo.com 


\section{INTRODUCCIÓN}

Las neuritis ópticas retrobulbares constituyen la inflamación retrobulbar del nervio óptico, siendo las enfermedades desmielinizantes su etiología más frecuente, y dentro de éstas, la esclerosis múltiple. Existen otras enfermedades que pueden dar lugar a un cuadros desmielinizantes como las infecciones, vasculitis etc. Recientemente, se ha demostrado que los inhibidores del factor de necrosis tumoral a $(\mathrm{TNF} \alpha)$ también pudieran ser causantes de neuritis ópticas (1).

\section{CASO CLÍNICO}

Mujer de 76 años de edad que acude de urgencia a nuestro hospital por presentar disminución brusca de la agudeza visual de su ojo izquierdo de 24 horas de evolución. Entre los antecedentes personales, destaca la presencia de una artritis reumatoide de larga evolución que había precisado tratamiento con Metotrexate y que en la actualidad estaba siendo tratada con Infliximab (Remicade ${ }^{\circledR}$ ) desde hacía 20 meses. No presentaba antecedentes oftalmológicos de interés.

La exploración oftalmológica inicial fue la siguiente: agudeza visual corregida ojo derecho (OD): 1,0 y ojo izquierdo (OI): 0,15 ; defecto pupilar aferente relativo en ojo izquierdo; la biomicroscopía del segmento anterior mostraba cataratas córtico-nucleares bilaterales; y en el fondo de ojo se apreciaban drusas duras sobre arcadas vasulares así como una mácula y nervio óptico de aspecto normal. Ante este cuadro se solicitó analítica y velocidad de sedimentación globular (VSG) de urgencias, que fueron normales, y se realizó una campimetría Humphrey 30-2 que mostró una depresión generalizada de la sensibilidad en ojo izquierdo, estando el campo visual derecho normal (fig. 1). El test de colores, Farnsworth-Munsell, del ojo izquierdo presentaba una alteración a nivel del eje azul amarillo.

Ante la sospecha de una lesión que afectara la vía visual prequiasmática izquierda, se solicita una resonancia nuclear magnética cráneo-orbitaria que muestra lesiones desmielinizantes múltiples de la sustancia blanca subcortical y periventricular con afectación de la unión calloso-septal (figs. 2 y 3 ). Teniendo en cuenta la morfología irregular de las lesiones, y su localización característica, y a pesar del criterio en contra de la edad, hizo que la escle-

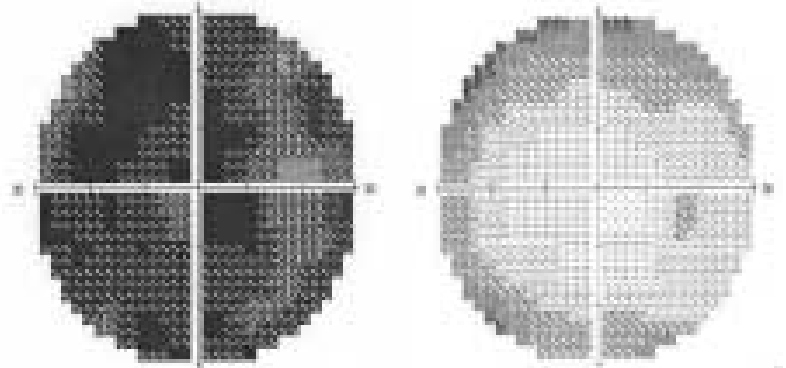

Fig. 1: Disminución generalizada de la sensibilidad en ojo izquierdo.

rosis múltiple fuera la primera posibilidad a tener en cuenta. Sin embargo, y tras realizar una interconsulta a neurología, se plantea la posibilidad de que se trate de un cuadro de neuritis óptica retrobulbar de etiología desmielinizante en relación con el tratamiento con Infliximab. Se decidió suspender el tratamiento con Infliximab e instaurar tratamiento con pulsos de $1 \mathrm{~g}$ de metilprednisolona durante 3 días, objetivándose una clara recuperación de la agudeza visual $(1,0)$ así como la desaparición del escotoma campimétrico en los sucesivos controles y hasta el momento actual (fig. 4).

\section{DISCUSIÓN}

El factor de necrosis tumoral TNF $\alpha$ es una citoquina derivada de los macrófagos que estimula los procesos inflamatorios mediante su unión a los

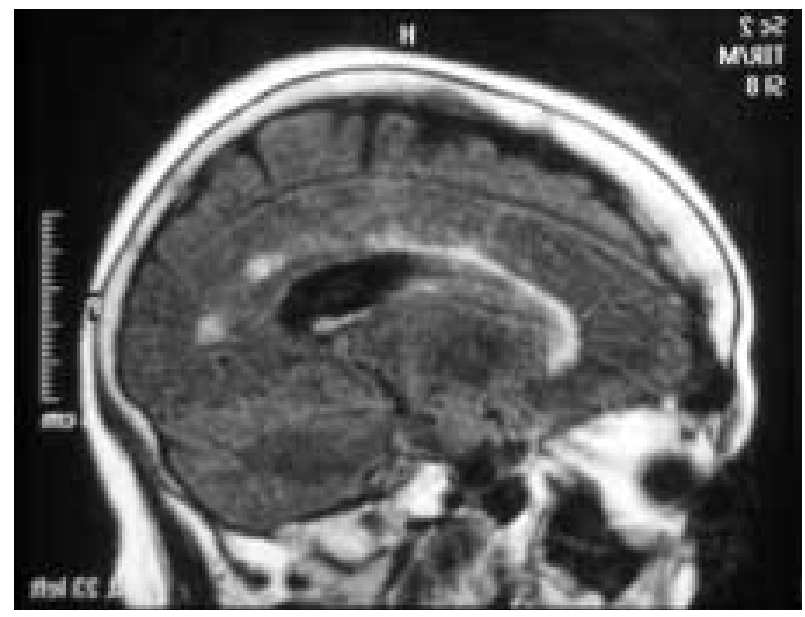

Fig. 2: Corte sagital de resonancia magnética que muestra placas de desmielinización periventricular características. 


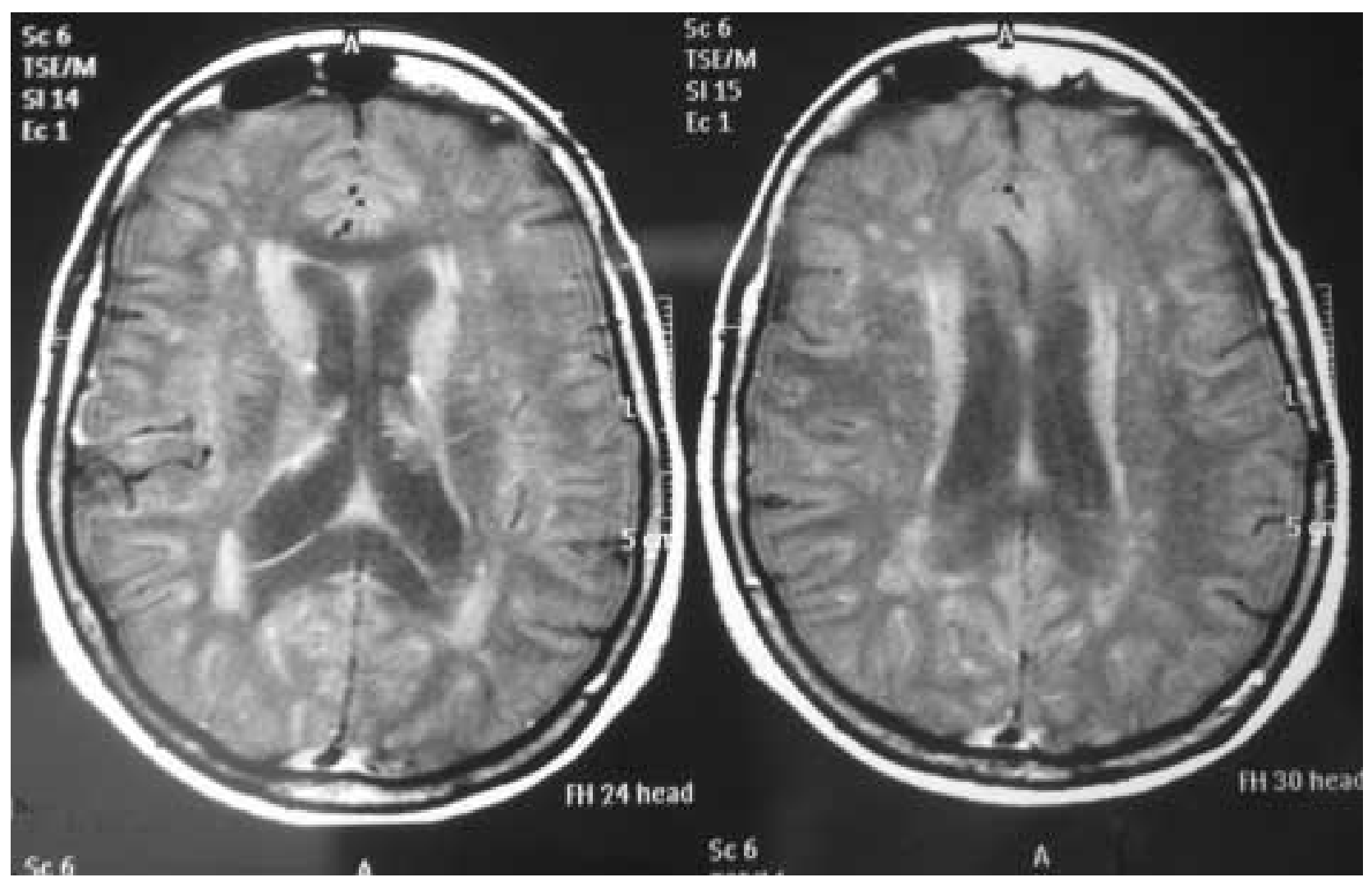

Fig. 3: Lesiones desmielinizantes en el corte transversal de la resonancia magnética.

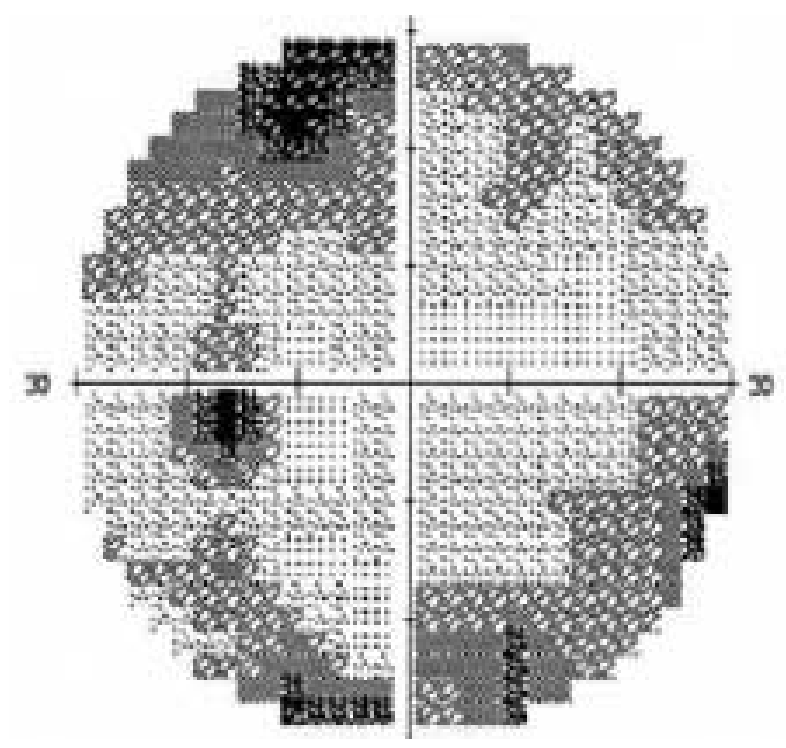

Fig. 4: Recuperación del campo visual izquierdo tras suspensión del tratamiento con Infliximab. receptores de superficie celular. Infliximab es un anticuerpo tipo IgG que se une al $\mathrm{TNF} \alpha$ inhibiendo su actividad.

Desde la introducción de los anti TNF $\alpha$ en el tratamiento de la artritis reumatoide y en la enfermedad de Crohn, su uso se ha extendido como tratamiento inmunomodulador de diferentes enfermedades autoinmunes hasta convertirse en una medicación de uso habitual entre estos pacientes.

A pesar de la alta seguridad que presenta este fármaco en pacientes diagnosticados de artritis reumatoide, se han descrito efectos adversos tanto respiratorios como neurológicos incluyendo dos casos de exacerbación de la desmielinización preexistente en pacientes tratados con infliximab y que previamente presentaban una esclerosis múltiple de progresión rápida (2). En el año 2002 Foroozan et al (1) describen el primer caso de neuritis óptica retrobulbar de etiología desmielinizante en una paciente de 55 años con artritis reumatoide y que 
estaba en tratamiento con Infliximab. A pesar de la posibilidad de que se pudiera tratar de un hallazgo casual, los autores enfatizan la necesidad de estar alerta ante la posibilidad de encontrarnos con eventos desmielinizantes en pacientes que estén siendo tratados con inhibidores del TNFo. Esta recomendación se sustenta en la similitud de este caso con los dos casos de exacerbación de una esclerosis múltiple tras el tratamiento con infliximab (2) y en los resultados obtenidos en ensayos con lenercept (otro inhibidor del TNF $\alpha$ ) en pacientes con esclerosis múltiple, donde se ha observado una mayor tendencia hacia la exacerbación de la enfermedad en estos pacientes en comparación con el grupo placebo (3). Posteriormente se han descrito nuevos casos de neuritis desmielinizante en pacientes con otras patologías (enfermedad de Crohn) que habían recibido tratamiento con Infliximab (4) así como algún caso de neuropatía óptica anterior bilateral relacionada con el tratamiento con Infliximab.

Debido a los factores etiopatogénicos que relacionan a los inhibidores del TNFo con la aparición de eventos desmielinizantes, así como la edad avanzada de nuestra paciente, a la cual un primer brote de esclerosis múltiple es muy infrecuente, consideramos que se trata de un caso de desmielinización asociada al tratamiento con Infliximab.
Para concluir, quisiéramos poner de manifiesto la posibilidad de encontrarnos en el futuro con cuadros similares al que presentamos y la necesidad de suspender la medicación con inhibidores del TNF alfa (5) en estos casos, debido a la creciente popularidad de estos fármacos en nuestros hospitales.

\section{BIBLIOGRAFÍA}

1. Foroozan R, Buono LM, Sergott RC, Savino PJ. Retrobulbar optic neuritis associated with infliximab. Arch Ophthalmol 2002; 120: 985-987.

2. van Oosten BW, Barkhof F, Truyen L, Boringa JB, Bertelsman $F W$, et al. Increased MRI activity and inmune reaction in two multiple esclerosis patients treated with the monoclonal anti-tumor necrosis factor antibody cA2. Neurology 1996; 47: 1531-1534.

3. The Lenercept Multiple Sclerosis Study Group and The University of British Columbia MS/MRI Analysis Group. TNF neutralization in MS: Results of a randomized, placebo-controled multicenter study. Neurology 1995; 53. 457-465.

4. Strong BY, Enry BC, Herzenberg H, Razzeca KJ. Retrobulbar optic neuritis associated with Infliximab in a patient with Crohn Disease. Ann Intern Med 2004; 20: 140: E678.

5. Rebolleda G. Infliximab y neuritis óptica. Arch Soc Esp Oftalmol 2004; 79: 591. 